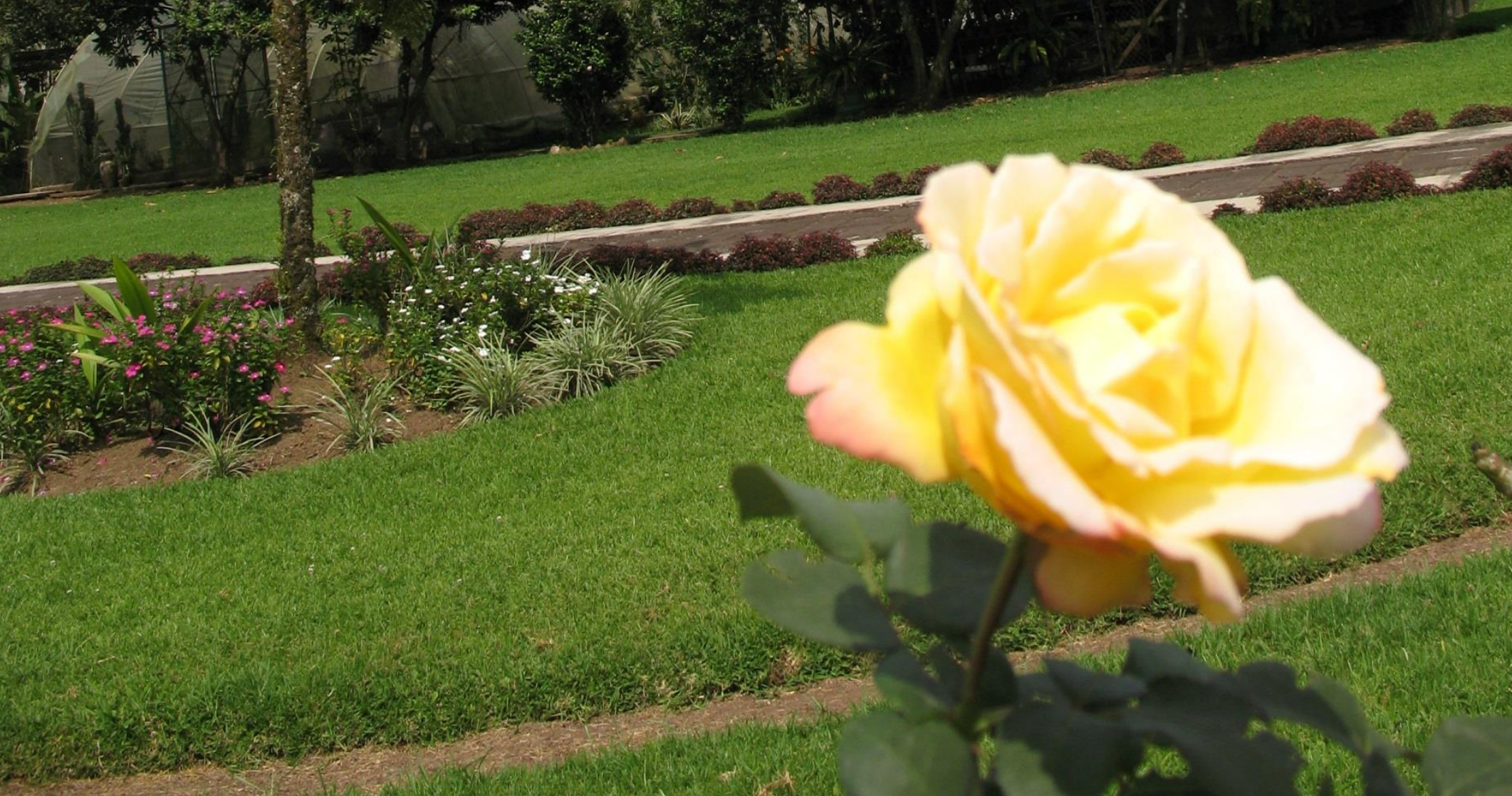

\title{
Iniciativa basada en la evidencia para regular la prescripción y venta de esteroides anabólicos androgénicos
}

José Luis Castillo Hernández - María Magdalena Álvarez Ramírez - Krystal Denicce González Fajardo -Edith Yolanda Romero Hernández - Susana Sánchez Viveros

RESUMEN: Se propone gestionar la aprobación de una iniciativa de Ley basada en la evidencia para regular la prescripción y venta de esteroides anabólicos androgénicos (EAA) a través de una Reforma a la Ley General de Salud en los artículos 17 Bis fracción VII al artículo 181, proponiendo emitir normas técnicas obligatorias o directivas generales, aplicables a los establecimientos cubiertos o descubiertos, destinados para la práctica de ejercicios o deporte. Las evidencias que sustentan dicha investigación se obtuvieron a través de un Estudio transversal descriptivo, en el cual se aplicó un instrumento en 15 gimnasios para fisicoconstructivismo en la ciudad de Xalapa, Ver. Entre otros datos importantes, se encontró prevalencias de $25 \%$ en el uso de EAA, los principales motivos para consumirlos fueron el aspecto personal (52\%) y el rendimiento físico (24\%). En la gran mayoría de los casos quien prescribió estas sustancias fue el entrenador (63\%) y en algunos casos algún compañero del mismo gimnasio. El 71\% de quienes dijeron consumir estas sustancias los adquieren en farmacias y un 29\% los consume de diseño. Este panorama representa un serio problema de salud pública por las implicaciones que representa el consumo de sustancias dopantes para el organismo, ya que los daños a la salud pueden ir desde cáncer de hígado y próstata, elevación de 
la presión arterial, acné, disturbios psicológicos, engrosamiento de la voz, arritmias del corazón, nerviosismo, perdida de la coordinación, conducta agresiva, problemas articulares y de ligamentos, caída del cabello, hasta desfiguración por crecimiento óseo excesivo

Palabras clave: Esteroides anabólicos; prevalencias; Iniciativa de Ley.

ABSTRACT: It is proposed to manage the approval of an initiative of Law based on evidence to regulate the prescription and sale of anabolic androgenic steroids (EAA) through a Reform to the General Law of Health in articles 17 Bis fraction VII to article 181, proposing issue mandatory technical norms or general directives, applicable to covered or discovered establishments, intended for the practice of exercises or sports. The evidence supporting this research was obtained through a descriptive cross-sectional study, in which an instrument was applied in 15 gyms for physicoconstructivism in the city of Xalapa, Ver. Among other important data, prevalences of $25 \%$ were found in the use of EAA, the main reasons for consuming them were the personal aspect (52\%) and the physical performance (24\%). In the vast majority of cases who prescribed these substances was the coach (63\%) and in some cases a partner of the same gym. $71 \%$ of those who said they consume these substances acquire them in pharmacies and 29\% consume them in design. This panorama represents a serious problem of public health due to the implications that the consumption of doping substances represents for the organism, since the damage to health can range from cancer of the liver and prostate, elevation of blood pressure, acne, psychological disturbances, thickening of the voice, arrhythmias of the heart, nervousness, loss of coordination, aggressive behavior, joint and ligament problems, hair loss, even disfigurement due to excessive bone growth.

Keywords: Anabolic Steroids; Prevalence; Initiative of Law.

\section{Introducción}

El dopaje, en muchas ocasiones, conlleva a la adicción a algún tipo de sustancia prohibida, lo cual puede provocar la muerte del usuario. Dentro de las sustancias dopantes se encuentran los esteroides anabólicos androgénicos (EAA), (o su nombre simplificado como esteroides anabolizantes), en la actualidad el abuso de toda clase de sustancias para mejorar el rendimiento deportivo y la forma física no es exclusivo de los deportistas de élite, sino que se ha extendido a las personas que acuden a gimnasios regularmente (Caba, 2006).

En 2006, tanto González, como Sánchez y Mataix, destacaban que entre los posibles cambios patológicos inducidos por los EAA se encuentran los riesgos sustanciales para la salud, incluyendo alteraciones en el metabolismo de las lipoproteínas, en el sistema reproductor, 
hepatotoxicidad y cardiotoxicidad. Sin embargo, no todos los esteroides producen los mismos efectos.

Yesalis (2000) señala que el problema en el consumo de EAA se ve agravado porque en muchos de los casos son los propios entrenadores quienes los proporcionan a los deportistas, tanto a nivel de deporte de alto rendimiento como en deportistas amateurs y recreativos. En los gimnasios se dice que su venta es cosa de todos los días. En los Estados Unidos solo se venden por prescripción médica, pero en México se pueden adquirir sin receta, y también se les puede hallar en las farmacias veterinarias con la ventaja de que son más baratos. Al no ser recetados por un médico, se recurre a la automedicación y se calcula que los consumidores se administran hasta cien veces la dosis recomendada.

El no contar con una ley que regule la prescripción y venta de Esteroides Anabólicos Androgénicos trae como consecuencia que estos se puedan adquirir sin ninguna dificultad y en consecuencia que su prescripción se realice por personas no calificadas o bien se auto prescriba, lo cual representa serios problemas para la salud. Si a lo anterior se agrega que los gimnasios para físico-constructivismo deben representar una opción para la práctica de ejercicio y sus beneficios para la salud y es ahí donde precisamente se prescriben, representan un riesgo para la salud. Con la realización de esta investigación se contó con evidencia para formular una iniciativa de Ley a fin de regular la prescripción y venta de Esteroides Anabólicos Androgénicos, misma que fue presentada a la Comisión de Salud de la H. Legislatura de San Lázaro para su análisis y en su caso, aprobación.

\section{Planteamiento del problema}

En México en materia de salud pública la falta de legislación para la regulación de venta de medicamentos, autoprescripción y automedicación representa un grave problema resultado de un inadecuado y débil sistema de salud. De acuerdo con la Organización Panamericana de Salud (OPS) la responsabilidad de la automedicación no recae en el paciente o consumidor, además recalca la falta de profesionalidad del sistema de comercio y la abierta infracción de las disposiciones legales por parte de las personas responsables de cumplirlas. La distinción de México frente a muchos otros países es la carencia de información que apoye el uso de medicación segura y responsable. Se adhiere el hecho de que la venta de medicamentos frecuentemente son expedidos sin necesidad de receta médica, esta autoprescripción es desalentada por la Organización Mundial de Salud (OMS) debido a que el uso de estos medicamentos requiere supervisión médica pues tienen un margen de riesgo/beneficio.

La Ley General de Salud (2016), artículo 221 define como medicamentos a: 
toda substancia o mezcla de substancias de origen natural o sintético que tenga efecto terapéutico, preventivo o rehabilitatorio, que se presente en forma farmacéutica y se identifique como tal por su actividad farmacológica, características físicas, químicas y biológicas. Cuando un producto contenga nutrimentos, será considerado como medicamento, siempre que se trate de un preparado que contenga de manera individual o asociada: vitaminas, minerales, electrólitos, aminoácidos o ácidos grasos, en concentraciones superiores a las de los alimentos naturales y además se presente en alguna forma farmacéutica definida y la indicación de uso contemple efectos terapéuticos, preventivos o rehabilitatorios.

Además el artículo 226 dice que todos los medicamentos necesitan receta médica para ser expedidos excepto aquellos que ofrecen tratar padecimientos menores y pueden ser adquiridos en farmacias (fracción V), y los que puedan ser expedidos en otros establecimientos que no sean farmacias (fracción VI); de acuerdo a la fracción IV algunos medicamentos podrán resurtirse tantas veces lo indique el medico que prescriba.

En el ejercicio y deporte el uso de suplementos y otras sustancias se ve motivado por personas insatisfechas con su aspecto físico que carecen además de orientación profesional adecuada y se ve facilitado por esta falta de legislación específica que prohíba la venta sin receta médica (Moura Lacerda, et al., 2015). Incluso con raras excepciones, los suplementos son innecesarios cuando la dieta de una persona es adecuada en calidad y cantidad y acompañada de una ingesta de líquidos adecuada apropiada.

El uso de dopantes en el deporte competitivo y en contextos no competitivos es evidente, datos comprobados sugieren que el uso de estos en la población de atletas y no atletas puede ser de 15\% con predominio en hombres. La agencia Mundial Antidopaje (AMA) define como dopaje a la detección y/o uso de sustancias prohibidas, metabolitos o los marcadores de esa sustancia en el cuerpo. Entre estos agentes dopantes se encuentran los Esteroides Anabólicos Androgénicos (EAA). (Bird, et al., 2015).

El listado actualizado de medicamentos de referencia (2016) publicado por la Comisión Federal para la Protección contra Riesgos Sanitarios (COFEPRIS) indica que el undeocanoato de testosterona y el enantato de testosterona en solución inyectable y capsulas, ambos EAA, necesitan de receta médica apegándose a esta última fracción y que no deberán de ser expedidos en libre venta.

Desde la década de 1970, los esteroides anabólicos androgénicos (EAA) han sido abusados a ritmos cada vez mayores en atletismo competitivo, en deportes recreativos y en culturismo (Nieschlag \& Vorona, 2015). Estos son variaciones sintéticas de la hormona sexual masculina testosterona sintetizada en los testículos por las células Leidig. El término anabólico se refiere a 
la formación de músculos mientras que andrógeno se refiere al aumento de las características sexuales masculinas o desarrollo estas.

Los Esteroides Anabólicos Androgénicos (EAA) producen reacciones adversas si se usan indiscriminadamente con la finalidad de aumentar la masa muscular o el rendimiento físico, se usan dosis altas durante tiempo prolongado y en combinación con otras sustancias (Vázquez Mourelle, et al., 2015), la dosis puede llegar hasta 100 veces lo recomendado, aunado el hecho de que se consume la versión veterinaria ya que resulta más barata (Castillo Hernández, et al., 2013) aunque estos esteroides veterinarios contienen los mismos componentes que los esteroides para uso humano no son tan puros.

La información sobre el uso de cualquier medicamento debe ser tratada desde la etapa educativa. La importancia de esto es mantener la ética deportiva que contiene un alto rigor de respeto en cuanto a las legislaciones vigentes acerca del consumo de sustancias y prácticas en atletas.

\section{Forma de presentación}

Los esteroides anabólicos vienen en diversas formas, incluidas pastillas, cremas, parches, tabletas, inyecciones o gotas que se ponen debajo de la lengua.

\section{Clasificación de EAA}

- Testosterona y derivados. Formas de presentación: Propionato, Suspensión, Sustanon 100, Sustanon 250, Omnadren, Sten, Test 400, Testovirón, Cipionato, Undecanoato, Enantato, Undecilinato de Boldenona, Metiltestosterona, Metandrostenolona.

- Dihidrotetosterona y derivado. Formas de presentación: Drostanolona, Oximetolona, Estanozolol, Metenolona, Oxandrolona, Etilestrenol, Testolactona, Dromostenolona.

- Nandrolona y derivados. Formas de presentación: Decanoato de Nandrolona, Fenylpropionato de Nandrolona, Noretandrolona, Etilestrenol, Trembolona, Laurabolin, Durabolín.

\section{Clasificación de EAA de uso veterinario}

Los EAA de uso veterinario fueron clasificados de acuerdo al nivel de riesgo por sus ingredientes activos, el grupo I está compuesto por los ingredientes activos de productos farmacéuticos veterinarios restringidos para venta mediante receta médica cuantificada y uso exclusivo del médico veterinario, su uso representa un riesgo de toxicidad para la sanidad animal, el grupo II 
lo conforman los ingredientes activos de productos farmacéuticos veterinarios que para su comercialización necesitan receta médica simple, pueden llegar a ser tóxicos si no se supervisa su empleo y en el grupo III se consideran los productos farmacéuticos veterinarios de venta libre en el país cuyos ingredientes activos son seguros (SAGARPA, 2004).

Dentro del grupo I se encuentran los anabólicos inyectables con efecto androgénico (undecilenato de boldenona, nandrolona, oxandrolona, metandrostenolona, metadiona, trembolona, estanazolol y methandianona) y en el grupo II los implantados con efecto androgénico (testosterona) lo que supone un riesgo para el consumo humano muy grande.

\section{Efecto en la pubertad}

Cuando el consumo se realiza previo o durante la pubertad se han reportado cierres prematuros de las epífisis y que pueden disminuir la estatura final, la hipertensión, el incremento en lipoproteínas de baja densidad (LDL) y la disminución en lipoproteínas de alta densidad (HDL). Puede contribuir al incremento en el riesgo de enfermedades cardiovasculares. (Contreras, 2015).

\section{Efectos por género}

En los hombres:

- Atrofia testicular

- Azoospermia

- Calvicie

- Ginecomastia

- Aumento en el riesgo de cáncer de la próstata

En las mujeres:

- Crecimiento de vello facial o vello corporal en exceso

- Patrones de calvicie masculina

- Parar o cambiar el ciclo menstrual

- Agrandamiento del clítoris

- Voz profunda

- Tamaño disminuido de las mamas

- Lívido aumentado o disminuido 


\section{Riesgos inmediatos}

En personas que usan EAA de forma inyectada aumenta considerablemente el riesgo de contraer o transmitir el Virus de la Inmunodeficiencia Humana (VIH) / Síndrome de Inmunodeficiencia Adquirida (SIDA) o el virus de la hepatitis.

\section{Efectos a corto y largo plazo}

A corto plazo pueden aparecer signos como celos paranoicos (extremos y sin razón), irritabilidad extrema, ilusiones-creencias o ideas falsas, criterio alterado edema en extremidades. A mediano y largo plazo está la aparición de acné, la ictericia que puede indicar disfunción hepática y que se vincula con el consumo de esteroides anabolizantes por vía oral, también pueden ocurrir lesiones musculotendinosas y disfunciones hepáticas o tumores (benignos y malignos), insuficiencia o problemas renales, acromegalia, presión alta, cambios en el colesterol sanguíneo, con lo cual aumenta el riesgo derrame cerebral y ataque del corazón incluso en personas jóvenes.

\section{Efectos psicológicos}

Cambios de humor, conducta irracional, incremento de la agresividad ("ira esteroidea"), irritabilidad, depresión y dependencia. El uso continuado de estas sustancias produce síndrome de abstinencia al interrumpir su uso y pueden implicar cambios notables en el estado de ánimo, fatiga, desasosiego, pérdida de apetito, insomnio, lívido reducido y deseos vehementes por los esteroides. Uno de los síntomas más peligroso es la depresión porque cuando persiste puede conllevar a intentos de suicidio.

En casos de mayor gravedad algunos cambios del estado mental reportados por diversos estudios que se asocian al uso de anabólicos incluyen paranoia, delirio, manías y tendencia homicida.

\section{Formas de administración}

- Ciclos: tomar dosis por un periodo de tiempo, parar por un tiempo y después volver a empezar a tomarlos

- Apilamiento: combinando dos o más tipos diferentes de los esteroides

- Pirámide: lentamente aumentando la dosis o frecuencia de abuso, llegando a una cantidad máxima, y después gradualmente reducir el consumo. 
En cuanto al ejercicio este es definido por la OMS como: "una variedad de actividad física planificada, estructurada, repetitiva y realizada con un objetivo relacionado con la mejora o el mantenimiento de uno o más componentes de la aptitud física."

\section{Otras consideraciones}

De los trastornos de la conducta se puede mencionar la dismorfia muscular en los que de acuerdo a los criterios de Pope et al. (1997), estas personas tienen gran preocupación por el tamaño corporal, levantan pesas de 3 a 7 días por semana, realizan dietas hiperproteicas y en ocasiones utilizan suplementos alimentarios y esteroides. La dismorfia muscular es un trastorno caracterizado por una preocupación excesiva con la idea de no tener un cuerpo suficientemente magro o musculoso, lo que conlleva renunciar a actividades sociales y laborales por la necesidad de ejercitarse (American Psychiatric Association, 2013). El riesgo de padecer este trastorno son aquellos deportistas que practican compulsivamente levantamiento de pesas o fisicoconstructivistas y se estima que un 10\% de estos los padecen (Pope, et al., 1997).

\section{Metodología}

- Se realizaron visitas a los gimnasios previamente seleccionados de la ciudad de Xalapa. De los gimnasios visitados, solo 15 aceptaron participar en el estudio. El número de participantes fue de 173 usuarios que cumplieron con los criterios de inclusión de acudir por lo menos 3 veces a la semana al gimnasio, durante un periodo no menor a un año de antigüedad.

- En el Laboratorio de Evaluación del Estado Nutricio de la Facultad de Nutrición Xalapa de la Universidad Veracruzana se evaluó la composición corporal y en el Laboratorio Análisis Clínicos de la Facultad de Bioanálisis fue tomada la muestra para la realización del perfil lipídico.

\section{Procesamiento y análisis de la información}

Los resultados del cuestionario fueron codificados en una base de datos elaborada a través del software statistica 9.0. Se realizó un análisis exploratorio de los datos a través de estadísticas descriptivas de variables en estudio: prevalencia generales y por sexo de dopaje con EAA, motivos para doparse, prescripción, etc. Se realizaron pruebas de estimación de asociación entre dopaje, escolaridad, situación en el trabajo y conocimientos sobre sus efectos. 


\section{Resultados}

1.- Los resultados obtenidos de esta investigación sirvieron de insumo como evidencia para proponer una Iniciativa de Ley.

2.- El Instituto de Investigaciones Jurídicas de la Universidad Veracruzana bajo la Dirección de la Dra. Petra Armenta Ramírez, fue el encargado de la elaboración de la Iniciativa de Ley.

3.- Dicha Iniciativa fue presentada en el Foro: Iniciativa basada en evidencia para regular la prescripción y venta de esteroides anabólicos androgénicos en México, realizado por el Cuerpo Académico UV-CA-437 Estilos de Vida Saludable, celebrado el 2 de Marzo del 2018 en la USBI Xalapa.

4.- Posteriormente, en Abril del 2018 fue entregada a la Comisión de Salud de la Cámara de Diputados de la LXIII Legislatura en el Palacio Legislativo de San Lázaro en la ciudad de México.

\section{Referencias}

Bird, S., Goebel, C., Burke, L. \& Greaves , R., 2015. Dopaje en el deporte y el ejercicio: problemas anabolicos, ergogénicos, de salud y clínicos. Revista Internacional de Medicina de Laboratorio, 17 Septiembre, 53(2), pp. 196-221.

Caba, M. (2006). Esteroides anabólicos: bonitos por fuera, muy malos por dentro. La Ciencia y El Hombre, 19(2). Recuperado de https://www.uv.mx/cienciahombre/revistae/vol19num2/articulos/anabolicos/

Castillo-Hernández, J. L., Sánchez-Collado, P., \& Romero-Hernández, E. Y. (2013). Esteroides anabólicos androgénicos en usuarios de gimnasios para físico-constructivismo de Xalapa, Veracruz. Medicina, Salud y Sociedad, 3(3). Recuperado de http://cienciasdelasaluduv.com/site/images/stories/3_3/01).pdf

COFEPRIS, 2016. Listado actualizado de medicamentos de referencia 2016/02. [En línea] Available at: https://www.gob.mx/cms/uploads/attachment/file/175881/LMR_2016 02_V002.pdf [Último acceso: 16 Agosto 2017].

Contreras-Caro, J.G. 2015.Esteroides Anabólicos. V19 097.976. República Bolivariana de Venezuela.

Diario Oficial de la Federación, 2016. Ley General de Salud, México: s.n.

González-Gallego, J., Sánchez-Collado, P., \& Mataix-Verdú, J. (2006). Nutrición en el deporte, ayudas ergogénicas y dopaje. España: Díaz de Santos. 
Moura Lacerda, F. M. y otros, 2015. factores asociados con el uso de suplementos dietéticos por personas que ejercen en gimnasios. Revista de Saúde Pública, Volumen 49, p. 49:63.

Nieschlag, E. \& Vorona, E., 2015. Dopaje con esteroides anabolicos androgenicos (AAS): Efectos adversos en órganos y funciones reproductivas.. Endocrine and Metabolic Disorders, 16(2), pp. 199-211.

Pope, J. y otros, 1997. Muscle Dysmorphia: An Underrecognized Form Of Body Dysmorphic Disorder. Psychosomatics, Volumen 28, p. 548:557.

SAGARPA, 2004. ACUERDO por el que se establece la clasificación y prescripción de los productos farmacéuticos veterinarios por el nivel de riesgo de sus ingredientes activos., México: Diario oficial de la Federación.

Vázquez Mourelle, R. y otros, 2015. Control de la dispensación de medicamentos esteroides anabolizantes androgénicos. Gaceta Sanitaria, 29(4), p. 4:7.

Yesalis, C. E. (2000). Anabolic steroids in sports and exercise (2nd ed.). Champaign, Estados Unidos: Human Kinetics Publishers. 